\title{
Miranda
}

Revue pluridisciplinaire du monde anglophone /

Multidisciplinary peer-reviewed journal on the English-

speaking world

$14 \mid 2017$

Early American Surrealisms, 1920-1940 / Parable Art

\section{AS - Aux sources des negro spirituals : l'expérience de Port Royal à travers Slave Songs of the United States (1867)}

\section{Franck Ferraty}

\section{OpenEdition}

\section{Journals}

Édition électronique

URL : http://journals.openedition.org/miranda/10162

DOI : 10.4000/miranda.10162

ISSN : 2108-6559

\section{Éditeur}

Université Toulouse - Jean Jaurès

\section{Référence électronique}

Franck Ferraty, «AS - Aux sources des negro spirituals : l'expérience de Port Royal à travers Slave Songs of the United States (1867) », Miranda [En ligne], 14 | 2017, mis en ligne le 18 avril 2017, consulté le 16 février 2021. URL : http://journals.openedition.org/miranda/10162 ; DOI : https://doi.org/ 10.4000/miranda.10162

Ce document a été généré automatiquement le 16 février 2021.

\section{cc)}

Miranda is licensed under a Creative Commons Attribution-NonCommercial-NoDerivatives 4.0 International License. 


\title{
AS - Aux sources des negro spirituals : l'expérience de Port Royal à travers Slave Songs of the United States (1867)
}

\author{
Franck Ferraty
}

1 L'étude raisonnée Slave Songs of the United States (1867) - conçue comme une enquête rigoureuse - fait date dans l'histoire de la musique afro-américaine, car elle constitue la première transcription de 136 negro spirituals réunis, choisis, présentés et annotés par un trio de musicologues nord-américains blancs, pionniers en la matière. Marchant sur leurs traces, nous avons voulu contextualiser et sonder cette source, en souligner les pleins mais aussi les creux.

2 Sur les chemins de Port Royal et des Sea Islands, se dessine une "partition » originale black and white, d'où affleure l'onde vertueuse de trois chercheurs en quête d'humanité sur fond de scientificité. ${ }^{1}$

3 Le 7 novembre 1861, soit quelques mois seulement après le début de la guerre de Sécession, Port Royal, ville confédérée de Caroline du Sud située sur la côte est des États-Unis, tombait aux mains des troupes de l'Union. Dix mille esclaves furent ainsi libérés. Le Gideon's Band, ${ }^{2}$ constitué de missionnaires et d'abolitionnistes, militait alors pour la création d'écoles gratuites - aidé en cela par l'American Missionary Society - et pour que le gouvernement fédéral offrît des terres aux nouveaux affranchis leur permettant d'accéder à un marché du travail libre. C'est dans ce contexte d'émancipation grandissante que trois auteurs américains, William Francis Allen (1830-1889), ${ }^{3}$ Charles Pickard Ware (1840-1921), ${ }^{4}$ Lucy McKim Garrison (1842-1877), ${ }^{5}$ entreprirent de réunir des negro spirituals dans un recueil intitulé Slave Songs of the United States (1867). Sur la route d'une ethnomusicologie naissante, ${ }^{6}$ ils allaient sillonner de féconds territoires chantés à la recherche d'une identité noire à la force libératrice. 
Ces chants, transcrits, ont été méthodiquement collectés dans le voisinage de Port Royal (comté de Beaufort) et des Sea Islands ${ }^{7}$ (île de St. Helena notamment). Il fallait, à travers l'exégèse de productions vocales éminemment expressives, redonner une dignité à des hommes, des femmes et des enfants goûtant à la liberté après des années d'asservissement. Entre 1863 et 1864, William Francis Allen et son épouse Mary firent œuvre de militantisme en dirigeant dans les îles de Caroline du Sud une école pour les esclaves nouvellement affranchis, lesquels découvraient ainsi les vertus d'un enseignement émancipateur. ${ }^{8}$

5 Montrer et démontrer le génie afro-américain, tel fut le credo des trois musicologues qui s'employèrent à rendre audibles les mélodies noires dans leur contexte sociolinguistique. Fondatrice, cette mission musicale le fut dans le sens où pour la première fois on s'intéressait à un corpus original - et originel - jusque-là ignoré, car issu d'une population servile longtemps considérée comme non humaine. Le succès auprès des Blancs - de certains spirituals particulièrement fervents assit la réussite de cette entreprise philanthropique qui s'était donnée pour but de ré-humaniser l'homme noir à travers sa voix, celle d'un peuple en marche, une voix collective forçant le respect et l'admiration.

6 Lucy McKim Garrison avait déjà amorcé le mouvement de revival en consacrant en 1862 un article au sujet et en publiant le 8 novembre de la même année dans le Dwight's Journal of Music deux negro spirituals, «Roll Jordan » [«Roule, Jourdain »] ( $\left.n^{\circ} 1\right)$ et «Poor Rosy» [« Pauvre Rosy »] ( $\left.n^{\circ} 8\right) . .^{9}$ Dans la même mouvance, un certain H.G. Spaulding, un an plus tard, offrit à un mensuel new yorkais quelques spécimens de chants afro-américains dont « $\mathrm{O}$, Lord, remember me » [« $\mathrm{O}$, Seigneur, souviens-toi de moi »] ( $\left.n^{\circ} 15\right)$ et « The Lonesome Valley » [« La vallée solitaire »] $\left(n^{\circ} 7\right)$.

7 Outre les publications mentionnées ci-dessus, les sources proviennent essentiellement d'une compilation due à Charles Pickard Ware qui, dans Slave Songs of the United States, rassembla des negro spirituals à Coffin's Point, sur l'île de St. Helena :

Nous [W. F. Allen, C. P. Ware et L. McK. Garrison] avons jugé préférable de faire de ce recueil [celui de C. P. Ware] dans son intégralité la base de notre travail ; y sont inclus tous les hymnes jusqu'au ${ }^{\circ} 43$. Ceux qui suivent [ceux de W. F. Allen], jusqu'au n ${ }^{\circ} 55$, ont été recueillis par mes soins dans les plantations du Capitaine John Fripp et les plantations avoisinantes, sur la même île (Allen, Ware et Garrison 3).

8 Il faut ajouter à ce corpus de nombreuses contributions (une vingtaine), dont celles, remarquées, de Mr Kane O'Donnel ( ${ }^{\circ} 66$ et 82), du lieutenant-colonel Charles Tyler Trowbridge $\left(n^{\circ} 26,49,58,61,63-65,121\right)$, et surtout du colonel Thomas Wentworth Higginson (1823-1911), ${ }^{10}$ lequel, par ses compétences musicales, son engagement jamais démenti pour la cause noire, sa collaboration bienveillante à l'égard de ce projet, a apporté sa pierre d'angle à l'édifice $\left(\mathrm{n}^{\circ} 49,58-65\right) .^{11}$

9 La difficulté à transcrire ces chants fut réelle, car ceux-ci posaient d'épineux problèmes d'écriture tant sur le plan mélodico-rythmique que linguistique. De par leur aspect improvisé et glissant, ils ne se laissaient apprivoiser que relativement, la recherche de l'exactitude en ce domaine constituant un horizon chimérique. S'en approcher restait toutefois possible, après avoir pris la précaution de rappeler la volatilité des paroles sujettes à de nombreuses variantes, le caractère interchangeable et enchâssé de certains refrains, l'inconfort à traduire en notes de musique des inflexions mélodiques insaisissables. Miss McKim Garrison écrit ainsi : 
Il est difficile d'exprimer tout le caractère de ces ballades chantées par les noirs avec de simples notes de musique. Les étranges effets gutturaux, les curieux effets rythmiques produits par les voix de solistes qui font chorus à intervalles irréguliers, tout cela semble presque aussi impossible à transcrire sur une partition que le chant des oiseaux ou les sons d'une harpe éolienne (Allen, Ware et Garrison 6).

10 L'entreprise se révéla audacieuse car elle jetait les bases d'une expertise pionnière ${ }^{12}$ qu'il serait - au regard des résultats engrangés - malvenu de frapper d'« impuissance » ou de «nullité ». ${ }^{13}$ Slave Songs of the United States a le mérite d'exister, offrant par làmême à la communauté scientifique un corpus non négligeable de 136 spirituals contextualisés et transcrits susceptibles de faire avancer la recherche $; ;^{14}$ il est le premier ouvrage à transcrire sur papier un patrimoine jusque-là véhiculé par la seule tradition orale. [...] Ces faits marquants sont les prémices d'un effort collectif de propagation qui passera par la diffusion de partitions lors des tournées américaines, et parfois européennes, de la plupart des groupes universitaires impliqués dans la mouvance des Jubilee Songs (Balen 70, 73).

11 Concernant la méthode de transcription, les auteurs se sont imposés de ne négliger aucune variante, qu'il s'agisse des mélodies ou des paroles. Pour ce qui est de l'orthographe, ils ont adopté la démarche du colonel Higginson :

Les mots seront transcrits ici, le plus fidèlement possible, dans leur dialecte d'origine ; et si l'orthographe semble parfois incohérente, ou les fautes d'orthographe insuffisantes, c'est parce que je [T.W. Higginson] n'ai pas pu coller davantage au message original (Allen, Ware et Garrison 21).

Dans leur volonté de réhabiliter ces chants et pour leur donner une plus grande audience, les trois musicologues - sur le modèle de ce que préconisait alors l'actrice et écrivaine anglaise Frances Anne Kemble - en appelèrent à un compositeur de talent capable de mettre en scène un opéra à succès intégrant quelques couplets et refrains noirs. En 1867, il était encore un peu tôt pour que se réalisât un tel projet. En revanche, plus tard, Antonín Dvořák, ${ }^{15}$ avec sa 9e Symphonie dite du Nouveau Monde (1893), et George Gerschwin, avec son opéra Porgy and Bess (1935), concrétiseraient ce souhait.

13 Abolitionnistes et humanistes dans l'âme, les auteurs insistent sur l'aspect métissé de ces productions vocales originales mêlant inextricablement cultures noire et blanche : les termes utilisés - en dépit des précautions prises - témoignent néanmoins de la persistance de catégories et de réflexes sémantiques marqués par deux siècles et demi d'esclavage et donc de suprématie raciale (elle-même sous-tendue par une culture dominante d'obédience anglo-saxonne). Le paradoxe d'une telle attitude tient en ces quelques mots: une musique "civilisée » conçue par des "barbares" ou "demibarbares ".

14 L'imbrication du profane et du sacré, consubstantielle au genre spiritual, conduit William Francis Allen à s'interroger sur les transferts possibles de mélodies « purement noires » vers une pratique religieuse. En l'absence d'étude approfondie - tout au moins à l'époque - sur les spécificités propres aux musiques ethniques d'Afrique, l'auteur avance l'hypothèse d'une authenticité du répertoire fondée sur un processus d'africanisation de la pensée religieuse européenne: ainsi, les exemples de negro spirituals les mieux «révélés ", c'est-à-dire les plus enracinés dans leur africanité, seraient à rechercher dans des chants non religieux : 

negro spirituals. On remarquera que les noirs ont eux-mêmes transféré les meilleurs de ces spécimens vers un usage religieux - je suppose que selon le principe de Mr Wesley «il n'est pas juste que le Diable possède tous les bons airs de musique ». Les leaders et prédicateurs n'ont pas trouvé ce changement difficile à négocier; ou du moins ils ont pris si peu de peine à le faire qu'on détecte très souvent l'affleurement du profane, révélant ainsi l'origine des cantiques les plus solennels, en dépit des meilleures intentions du poète et de l'artiste (Allen, Ware et Garrison 21).

L'appropriation de l'hymnodie et des cantiques traditionnels protestants s'est faite par acculturation: les Noirs se sont convertis progressivement - avec plus ou moins de réticence de la part des planteurs exploitants - à la religion des Blancs et en se convertissant, ils ont adopté la langue des maîtres, c'est-à-dire l'anglais, qu'ils ont arrangé en «patois » : ils ont, de ce fait, créolisé le chanté européen en lui insufflant un « esprit » noir (goût pour le « shout ", l'improvisation, la transe). Les paroles faisaient l'objet très fréquemment de modifications liées aux aléas de l'improvisation collective, les Afro-américains prenant un malin plaisir à se réapproprier la langue des maîtres en arrangeant le texte à leur convenance, soit par analogie phonétique, soit pour faire coller coûte que coûte les mots à la mélodie, soit par incompréhension du sens, soit pour toutes ces raisons à la fois.

Il y a une spécificité du parler noir des îles de Port Royal liée à l'isolement de cette région. Forts de ce constat, les trois auteurs se livrent à une analyse sociolinguistique des idiomes afro-américains concernés apparus par acculturation, processus qu'ils qualifient de "décadence phonétique » et dont les termes trahissent curieusement un complexe de supériorité amoindri par l'usage des guillemets : une étymologie et une syntaxe réduites à leur plus simple expression, tels sont les corollaires de cette langue « indigène ». Il est coutume d'entendre les sons anglais th, $v$ ou $f$ atténués en $d$ et $b$ : «Chez ce peuple, le processus de "décadence phonétique» semble être allé probablement le plus loin qu'il puisse aller, et avec lui une extrême simplification de l'étymologie et de la syntaxe. Il y a, bien sûr, le traditionnel adoucissement des sons th, $\mathrm{v}$ ou $\mathrm{f}$ en $\mathrm{d}$ et $\mathrm{b}$; de même existe-t-il une fréquente permutation entre $\mathrm{v}$ et $\mathrm{w}$ comme dans veeds et vell pour weeds [mauvaises herbes] et well, woices et punkin wine pour voices et pumpkin vine [plantation de citrouilles]. «De wile (vilest) sinner may return » [ «L pécheur le plus vil peut revenir »] ( $\left.n^{\circ} 48\right)$. Ce dernier exemple illustre aussi cette habitude récurrente consistant à avaler les mots et les syllabes, comme lee'bro pour little brother, plänt'shun pour plantation » (Allen, Ware et Garrison 25).

Pour relativiser la portée "universelle» de ces productions vocales, les auteurs insistent sur le fait que les chants collectés l'ont été sur un périmètre étroit, les 43 premiers negro spirituals du recueil ayant été entendus sur une seule et même plantation, dont toutes les ressources musicales de surcroît n'avaient pas été épuisées. À cette réserve, il faut ajouter un autre obstacle potentiel, la réticence fort probable des Noirs nouvellement affranchis à chanter des airs qui leur rappelaient les temps sombres et douloureux de l'esclavage.

19 Les chants se déplaçant lentement, il n'était pas rare de trouver de multiples variantes d'une même mélodie cantonnées à un secteur restreint, tel Coffin's Point, plantation de coton située dans le voisinage de Port Royal, où convergèrent certains spirituals. ${ }^{16}$ Ainsi, à un mille de distance, on ne s'exprimait déjà plus tout à fait pareil (un « patois » par plantation) : pour preuve, l'identification de l'origine géographique de certains 
habitants établie grâce à leur parler. Celui-ci, dans sa structure interne, laissait transparaître les traces de la langue maternelle (dialecte africain) et de la langue dominante (anglais), le mélange et le métissage des deux aboutissant à un résultat curieusement étranger, c'est-à-dire non anglais : ${ }^{17}$

Les mots étranges, les curieuses manières de prononcer, les fréquentes abréviations, travestissent les traits familiers d'une langue maternelle, pendant que les modulations rythmiques, si caractéristiques de certaines langues européennes, lui donnent une sonorité entièrement non-anglaise. Après avoir passé six mois parmi eux, je [W. F. Allen] ne comprenais toujours pas certains élèves de mon école, pourtant parmi les plus assidus, sauf si par hasard ils parlaient très lentement (Allen, Ware et Garrison 24). Lucy McKim Garrison fut la première à identifier la «langue Gullah parlée par les exesclaves des Sea Islands, qui étaient venus d'Afrique tous en même temps et avaient conservé en partie leur dialecte d'origine, qu'ils mélangeaient avec l'anglais " (Hope Bacon 6). Il arrivait aussi que certains chants et leurs variantes fussent entendus dans des États limitrophes: le commerce d'esclaves entre plantations voisines facilitait les échanges permettant aux spirituals de circuler et de franchir aisément les frontières.

Pour se soustraire à la surveillance des maîtres, les esclaves se retrouvaient clandestinement la nuit dans les hush harbors (havres de paix ou abris silencieux) ou brush arbors (tonnelles de broussailles). Lors de ces réunions de grande ferveur religieuse, remontant à la surface, le corps possédé par l'esprit laissait affleurer les racines africaines via la danse, la transe et les cris qui en sont caractéristiques (Rouget 215-217). William Francis Allen nous donne une excellente description de ces «scènes de la forêt ", dont le but était de faire descendre l'Esprit afin que l'élu «trouve une chose », la foi en l'occurrence :

Une de leurs coutumes, à laquelle il est souvent fait allusion dans les chansons (comme dans le $\mathrm{n}^{\circ} 19$ ), est de déambuler à travers bois et marécages sous l'effet de l'exaltation religieuse, comme les bacchantes dans l'antiquité. Le sentiment religieux consiste pour eux à « trouver une chose (Allen, Ware et Garrison 12).

22 Le cri, résurgence de l'Afrique ancestrale - mais aussi imitation du pow-wow amérindien,$-^{18} \mathrm{a}$ frappé les esprits car il est l'incarnation fulgurante d'une expression vocale communautaire pulsionnelle (un exutoire aux traumatismes vécus). Il a élu domicile, entre autres, en Caroline du Sud (notamment à Port Royal) et dans les états situés au sud de celui-ci (en Floride en particulier). Il fut l'apanage des églises baptistes fort répandues dans les Sea Islands, où sa pratique se révéla féconde.

23 Les cris et les chansons de rameurs se partageaient les mêmes airs. La dimension spiritual s'invite tout particulièrement dans un chant de bateliers "Michael row the boat ashore" ["Michel rame vers la rive»] ( $\left.{ }^{\circ} 31\right)$, où le narrateur invoque directement l'archange Michel. Charles Pickard Ware a transcrit toute une série d'airs «marins » en respectant le tempo et le timing imposés par la succession régulière des coups de rame dans l'eau. Ces chants, profanes pour la plupart, entendus à Philadelphie et Baltimore, sur le Mississippi, ou encore à Port Royal, révèlent leur lien de parenté parfois étroit avec le continent africain: "Mr E.S. Philbrick a été frappé par la ressemblance entre certains airs chantés par les rameurs à Port Royal et les chansons des bateliers qu'il avait entendues sur le Nil » (Allen, Ware et Garrison 8). d'âme distincts obligeait les interprètes à varier les tempi en fonction des tâches qui 
leur étaient assignées : un même chant, comme par exemple "Poor Rosy ", interprété par des bateliers en train de ramer, par des enfants faisant tourner une meule, ou encore par une mère endeuillée, ne possédait pas le même tempo ni le même ethos.

Le colonel T.W. Higginson relate la manière dont un rameur lui expliqua comment il avait inventé une chanson (Higginson 692) : quelques mots mis bout à bout narrent une histoire, laquelle, vocalisée sur un air improvisé doté d'un refrain facilement mémorisable, est reprise en chœur par les autres rameurs :

Je [Higginson] me suis toujours demandé si elles [les chansons] avaient une origine consciemment définie, mentalement structurée, ou si elles se formaient par couches successives, par un processus quasi inconscient. Sur ce point, je ne pus recueillir aucun renseignement, bien que je posasse maintes questions, jusqu'à ce qu'enfin, un jour où je faisais la traversée en bateau de Beaufort à Ladies' Island, je me trouvai pour mon plus grand bonheur véritablement sur le chemin d'une chanson. [...] Mon rêve se réalisait, j'avais dépisté non seulement le poème mais aussi le poète. [...] Il se mit alors à chanter et les autres hommes, après l'avoir écouté un instant, reprirent en chœur le refrain comme s'ils le connaissaient depuis longtemps alors que de toute évidence ils ne l'avaient jamais entendu. Je vis avec quelle facilité une nouvelle « chanson » pouvait naître chez eux (Allen, Ware et Garrison 17-18).

Chose curieuse, W. F. Allen, C. P. Ware et L. McK. Garrison semblent convaincus que "les noirs n'ont pas de chants à plusieurs voix » (21-22) : ce constat ne colle pas à la réalité puisque les spirituals étaient chantés en polyphonie - certes réduite -, mais polyphonie tout de même. Peut-être faut-il voir dans cette erreur d'appréciation une difficulté à percevoir des lignes distinctes à l'intérieur de superpositions vocales relativement complexes, ${ }^{19}$ où la mélodie pouvait néanmoins primer sur l'harmonie en certains endroits (comme les refrains par exemple). Les auteurs d'ailleurs se contredisent un peu plus loin lorsqu'ils observent l'habitude des Noirs à ne pas " chanter la même chose » (5) ou à chanter de manière décalée - c'est-à-dire à plusieurs voix : «On chante en décalage, comme nous l'avons déjà mentionné, à tel point qu'à aucun moment il n'y a de pause complète. $C^{\prime}$ est dans «A House in Paradise » $\left(n^{\circ} 40\right)$ que ce décalage est le plus marqué » (23). Cette distorsion - involontaire - relevée dans le discours trahit néanmoins une non infaillibilité à l'égard du travail d'investigation entrepris, non infaillibilité d'ailleurs pleinement revendiquée et assumée par les trois musicologues qui, avec une modestie touchante, admettent une certaine marge d'« imperfection » dans leur action :

Conscients que celui-ci [le présent volume] comporte de nombreuses imperfections, qu'il est le résultat d'un travail de coopération considérable pendant près d'un an, les Auteurs le soumettent cependant au jugement du public, avec la conviction qu'il sera considéré comme digne d'être définitivement préservé (Allen, Ware et Garrison 38). Le titre Slave Songs of the United States - ambitieux - s'avère néanmoins abusif, car il semble sous-entendre que les auteurs ont pu arpenter la totalité des États-Unis. Une telle attitude a pu servir, plus ou moins consciemment, la propagande gouvernementale qui voyait là un moyen de diffuser l'image d'un grand pays réunifié à travers une étude ethnomusicologique de tout premier plan.

En réalité, l'ouvrage concernait, plus modestement, un nombre limité de chants collectés pour la plupart dans la région de Port Royal, ce dont étaient parfaitement conscients les auteurs qui reconnaissaient que les particularités rencontrées sur le 
terrain - élargies aux États esclavagistes du Sud-Est - ne pouvaient être érigées en règle générale.

Publiée en 1867, soit au lendemain d'une guerre fratricide, où les belligérants s'étaient férocement affrontés durant quatre longues années sur une ligne de fracture béante (celle de la Sécession), cette étude - engagée - se voulait d'abord et avant tout un manifeste en faveur de la politique d'émancipation menée par les troupes de l'Union sorties victorieuses du conflit et soucieuses de réconcilier le pays au sein de ce que l'on appelait alors le gouvernement fédéral. Il fallait affirmer énergiquement la cohésion politique d'un tel projet. Le recueil Slave Songs of the United States y contribue par sa volonté militante de traduire en musique les thèses abolitionnistes défendues par la nouvelle Union: les esclaves affranchis furent - pour un temps - élevés au rang d'humains regagnant ainsi une dignité perdue autrefois. Leurs spirituals participent à la révélation du génie musical noir, lequel clame haut et fort les couleurs de l'âme afroaméricaine.

Allen, William Francis, Pickard Ware, Charles et Lucy McKim Garrison. Slave Songs of the United States. New York : A. Simpson \& Co., Agathynian Press, 1867.

Allen, William Francis, Pickard Ware, Charles et Lucy McKim Garrison. Chants d'esclaves des États-Unis (traduction française : Francis Daubas, préface : Franck Ferraty).

Perpignan : éditions de Saint-Amans, 2015.

Balen, Noël. Histoire du Negro spiritual et du Gospel, de l'exode à la résurrection. Paris : Fayard, 2001.

Boorstine, Daniel. Histoire des Américains. Paris : Robert Laffont, 1991.

Chase, Gilbert. Musique de l'Amérique - America's Music, From the Pilgrims to the present (traduction française : Clara Babelon-Brooke). Paris : Buchet-Chastel, 1957.

Chenu, Bruno. Le grand livre des Negro Spirituals. Paris : Bayard, 2000.

Hope Bacon, Margaret. "Lucy McKim Garrison Pioneer in Folk Music". Pennsylvania History : A Journal of Mid-Atlantic Studies, Volume 54, $\mathrm{n}^{\circ} 1$ (January 1987) : 1-16.

Kemble, Frances Anne. Journal of a Residence on a Georgia Plantation in 1838-1839. Athens : University of Georgia Press, 1984.

Langel, René. Le Jazz orphelin de l'Afrique. Paris : Payot, 2001.

Ross, Alex. The Rest is Noise, À l'écoute du xxe siècle, La modernité en musique (traduction française de Laurent Slaars). Arles : Actes Sud, 2010.

Rouget, Gilbert. La Musique et la transe. Paris : Gallimard, 1990.

Stewart, Bruce E. « Port Royal experiment », Encyclopedia of Reconstruction Era, tome 2. Westport: Greenwood Press, 2006.

Wentworth Higginson, Thomas. "Negro Spirituals". The Atlantic Monthly, Volume 19, $\mathrm{n}^{\circ} 116$ (June 1867) : 685-694.

Yourcenar, Marguerite. Fleuve profond, sombre rivière. Paris : Gallimard, 1966.

Discographie

Gates, Rev J. M. Complete Recorded Works In Chronological Order (April to September 1926), volume 1. Document Records, DOCD-5414, 1995.

Gates, Rev J. M. Complete Recorded Works In Chronological Order (August 1934 to October 1941), volume 9. Document Records, DOCD-5484, 1996.

Lomax, Alan. Negro Prison Blues and Songs (recorded live at the Mississippi and Louisiana States Penitentiaries, 1947-1948). Legacy International, CD 326, 1994. 


\section{NOTES}

1. Je remercie chaleureusement Francis Daubas, professeur d'anglais retraité, de m'avoir fait l'amitié de traduire avec beaucoup d'à-propos l'ouvrage dont il est question ici, corpus de première main dont il faut souligner la qualité et la valeur historiographiques. Il a également traduit, pour les besoins de la présente étude, certains articles en américain figurant en bibliographie pour lesquels il n'existait pas de traduction préalable, ainsi que les jaquettes des CD mentionnés dans la discographie.

2. «Le Gideon's Band [...] développe un argumentaire marqué par un paternalisme bienveillant. Les Gideonites soutiennent que l'esclavage a infantilisé et durement affecté les esclaves sur le plan moral, de telle sorte qu'ils ne seront pas en mesure de faire face seuls aux lois du marché et à un environnement compétitif » (Stewart 487).

3. Né à Northbourough dans le Massachusetts, il étudia à Gottingen, Berlin et Rome. Il rentra en Amérique en 1856 et devint directeur adjoint de l'English and Classical School à West Newton (Massachusetts). Après la guerre de Sécession, il fut professeur en langues orientales et en histoire à l'Université de Wisconsin-Madison.

4. Cousin de William Francis Allen, il était éducateur et transcripteur de musique. Abolitionniste, il servit comme administrateur civil dans l'armée de l'Union, où il s'occupa avec son épouse Harriet d'esclaves affranchis dans les plantations de Port Royal pendant la guerre de Sécession.

5. Son travail à Port Royal constitue la première tentative de décrire les caractéristiques des negro spirituals : «En 1862, une jeune femme de dix-neuf ans, Lucy McKim Garrison, enquête plus systématiquement dans les Sea Islands, ces îles qui bordent la côte de Caroline du Sud et ont permis aux esclaves de mener une vie plus autonome» (Chenu 168). Par son engagement personnel - grâce aussi à la pugnacité de son mari Wendell -, elle vit son nom figurer comme auteur : «Après un printemps et un été consacrés à recueillir des chants, Lucy et Charles Ware relurent le manuscrit à l'automne et le livre fut publié en novembre 1867 . Wendell joua un rôle considérable dans la finalisation de l'ouvrage, mais en tant que mari amoureux et féministe de la première heure, il insista pour que le nom de l'auteur soit celui de Lucy, au même titre que William Francis Allen et Charles Pickard Ware » (Hope Bacon 12 ; traduction de Francis Daubas).

6. D'autres expériences similaires verraient le jour : Felipe Pedrell (1841-1922) parcourant l'Espagne pour recueillir des mélodies populaires; Béla Bartók (1881-1945) et Zoltán Kodály (1882-1967) recueillant le folklore hongrois (Structure strophique dans le chant traditionnel hongrois, 1906).

7. Les Sea Islands sont une série d'îles qui bordent le littoral de la Caroline du Sud, de la Géorgie et du nord-est de la Floride. Miss McKim en visita quatre au printemps 1862 : Hilton Head, St. Helena, Ladies, Port Royal. «En arrivant à Hilton Head, elle entendit pour la première fois les «tristes chants sauvages des Noirs». Elle commença à relever les paroles et la musique des «spirituals» (Hope Bacon 6 ; traduction de Francis Daubas).

8. «L'alphabétisation, à laquelle les affranchis furent les premiers à goûter, ne pouvait que donner des ailes et des armes à la volonté d'émancipation » (Langel 92).

9. En réalité, il ne s'agit pas du n 8 mais du n 9 si l'on s'en réfère à la nomenclature de l'édition originale.

10. Cet Américain fut un pasteur blanc unitarien et un fervent abolitionniste. En 1854, il mena l'assaut contre le palais de justice de Boston où était détenu Anthony Burns, un esclave fugitif devenu pasteur. Durant la guerre de Sécession, T.W. Higginson servit comme colonel du First South Carolina Volunteers, le premier régiment noir autorisé par le pouvoir fédéral mais dont les officiers étaient blancs. Ce régiment composé d'esclaves échappés de Caroline du Sud et de Floride fut actif du 31 janvier 1863 au 8 février 1864. En 1870, Higginson publia un ouvrage Army 
Life in a Black Regiment, où il relatait ses années d'officier passées auprès de soldats noirs. Le chapitre 9 intitulé «Negro Spirituals» constitue une source de premier plan quant à la connaissance des chants d'esclaves : il fut publié en « avant-première » dans l'Atlantic Monthly 19, $\mathrm{n}^{\circ}$ 116, daté de juin 1867. Sitôt paru, Higginson, en chercheur avisé et solidaire, mit généreusement son article à la disposition des trois musicologues, lesquels reconnaissaient avoir pu travailler sur les deux tiers du corpus, le tiers restant demeurant sans musique. «Fort lettré, amateur de poésie populaire européenne, ami d'Emily Dickinson, Thomas W. Higginson était particulièrement à même d'apprécier la puissance dramatique de leur patois barbare [celui de ses soldats noirs] et d'accepter certains illogismes typiques de la pensée primitive africaine, qui choquaient l'auditeur moyen du XIXe siècle beaucoup plus qu'ils ne nous choquent aujourd'hui » (Yourcenar 33).

11. «Ils [les auteurs] remercient plus particulièrement le colonel T.W. HIGGINSON pour ses encouragements amicaux et sa contribution directe ou indirecte à leur stock de chansons [...]. Il est peu de dire que sans sa coopération, cette Lyra Africana aurait perdu beaucoup de sa valeur. À travers lui nous avons profité de la chaleureuse assistance de Mrs. CHARLES J. BOWEN, Lieut.Colonel C. T. TROWBRIDGE, Capt. JAMES S. ROGERS, Rev. HORACE JAMES, Capt. GEO. S. BARTON, Miss LUCY GIBBONS, Mr. WILLIAM A. BAKER, Mr. T. E. RUGGLES, and Mr. JAMES SCHOULER » (Allen, Ware et Garrison 37).

12. «Par ses commentaires sur les chants d'esclaves des Sea Islands pendant la guerre de Sécession, et plus tard par l'aide qu'elle [Lucy McKim Garrison] apporta à leur publication, elle réalisa un travail de pionnière pour la diffusion des negro spirituals dans le domaine public » (Hope Bacon 1 ; traduction de Francis Daubas).

13. «En l'absence de tout moyen d'enregistrement, les observateurs s'efforcèrent de mettre en écriture les chants et danses qu'ils surprirent. Pour avouer finalement leur impuissance à transcrire des mélismes, des ornements que l'écriture occidentale ignorait. Ce ne sont donc que des intervalles et leur succession en mélodie qui témoignent de cette musique. On sait de surcroît la difficulté à mémoriser et à transcrire ensuite une musique entendue pour la première et unique fois [...]. Autant d'obstacles qui frappent de nullité la seule recherche qui aurait pu nous éclairer sur la négritude américaine de la fin du XIXe siècle » (Langel 130).

14. « [...] il [l'ouvrage Slave Songs of the United States] reste important comme source d'information » (Chase 187, 190).

15. «À New York [en 1892], il se lia d'amitié avec le jeune chanteur et compositeur noir Harry T. Burleigh, lequel lui fit découvrir l'univers des Negro Spirituals, qui, pour le vieux compositeur, contenait en germe l'avenir de la musique américaine. Il se lança rapidement dans la composition d'une Symphonie du Nouveau Monde, dans laquelle il mettait à profit quantité de thèmes mélodiques issus des traditions noire ou amérindienne " (Ross 176).

16. «Pour illustrer la lenteur avec laquelle ces chansons se déplacent on peut remarquer que "The Graveyard » ["Le cimetière"] ( $\left.\mathrm{n}^{\circ} 21\right)$ qui se chantait fréquemment dans la plantation du capitaine John Fripp pendant l'hiver 1863-64 n'atteignit pas Coffin's Point (distant de cinq milles) avant le printemps suivant. J'ai [W. F. Allen] entendu moi-même cette chanson à Pine Grove, deux milles plus loin, au mois de mars. Quelque part sur le trajet, cet air subit une forte transformation, comme on le verra d'après la variante donnée, qui est la forme sous laquelle j'avais l'habitude de l'entendre. Les numéros 38, 41, 42, 43, 118, 119, 122, 123 furent amenés à Coffin's Point après le départ de Mr Ware, par des réfugiés retournant à la plantation depuis la «ville » et le continent. De la même façon le $n^{\circ} 74$, « Nobody knows the trouble I see », qui était célèbre à Charleston en 1865, a depuis été apporté à Coffin's Point, avec très peu de transformations » (Allen, Ware et Garrison 12).

17. Ce juste constat devait rencontrer beaucoup plus tard un écho assez direct - bien que tout à fait fortuit - auprès de Daniel Boorstine et René Langel : « [...] dans la région côtière de la Caroline du Sud et de la Géorgie, des Noirs parlent un dialecte dit gullah dont le vocabulaire est 
composé de mots d'une vingtaine de langues africaines. Daniel Boorstine l'explique ainsi : « ... un nombre considérable d'esclaves était né en Afrique, et il en arrivait davantage chaque année. En 1858, soit un demi-siècle après que le commerce des esclaves africains eut été rendu illégal, un chargement de quatre cent vingt Noirs venus d'Afrique fut débarqué dans un port de Géorgie. La confusion des langues dut rappeler Babel! Comme les maîtres ne voulaient pas apprendre la langue de leurs esclaves, ceux-ci, souvent incapables de communiquer entre eux dans leur langue maternelle, se virent contraints d'apprendre ou d'inventer un jargon afin de se faire comprendre » (Langel 98-99, citant Boorstine).

18. «[...] le pow-wow se présentait en modèle que Blancs et Noirs ne manquèrent pas d'intérioriser soit par imitation, soit par dérision : la secte des trembleurs notamment et le rituel des camps de réveil, qu'elle inspira par conséquent. Les shouts de la première Église noire invisible, faits de danses marchées en rond, à pieds traînés, trouve dans son environnement le plus proche, dans la contingence, ce qu'on croyait hérité de la lointaine Afrique " (Langel 233-234).

19. «Le vrai «spiritual» est une création complexe et infiniment variée qui n'existe authentiquement qu'au moment où il est chanté » (Chase 190).

\section{RÉSUMÉS}

Le recueil Slave Songs of The United States (1867) constitue la première véritable contribution ethnomusicologique à l'exégèse des negro spirituals. Les auteurs - trois Nord-Américains d'origine blanche dont une jeune femme - ont collecté un ensemble de 136 chants qu'ils ont transcrits et annotés. Enquête rigoureuse s'il en fut, publiée à New York, elle posait les bases d'un corpus pionnier susceptible de faire avancer la recherche. Le présent article tente d'étudier et d'analyser cette source. Sur les chemins de Port Royal et des Sea Islands, se dessine une «partition » originale en noir et blanc d'où affleure l'onde vertueuse d'un trio de chercheurs en quête d'humanité sur fond de scientificité.

The collection Slave Songs of The United States (1867) constitutes the first true ethnomusicological contribution to the exegesis of negro-spirituals. The authors - three white North Americans, including a young woman - have collected 136 songs that they have transcribed and annotated. As an accurate enquiry, published in New York, it laid the foundations of an original corpus susceptible to make research progress. The present article attempts to examine and analyse that source. On the way to Port Royal and the Sea Islands, an original black and white «score " becomes apparent, to the surface of which rises the "virtuous wave » of three researchers in quest of humanity with a background of scientificity.

\section{INDEX}

Thèmes : Music

Mots-clés : émancipation, esclavage, ethnomusicologie, negro spiritual, transcription

Keywords : emancipation, ethnomusicology, negro spiritual, slavery, transcription 
AUTEURS

FRANCK FERRATY

Docteur en Musicologie

franck.ferraty@wanadoo.fr 